\title{
Repetitive trauma-induced extra-nuchal-type fibroma
}

\author{
Prem Ruben Jayaram ${ }^{1}$ (D) John Walsh ${ }^{1} \cdot$ Hamzah Lari $^{1} \cdot$ Nissreen Mohammad $^{2} \cdot$ Paul Ian Mallinson $^{1}$. \\ Hugue A. Ouellette ${ }^{1}$
}

Received: 16 September 2020 / Revised: 15 September 2021 / Accepted: 16 September 2021 / Published online: 23 September 2021 (C) ISS 2021

\begin{abstract}
A nuchal-type fibroma is a rare, benign fibrous tumour that typically occurs in the posterior neck along the midline, but can occur in extra-nuchal locations, most commonly in the back, shoulder and face. We present a biopsy-proven case that arose as a result of heavy gym-related activities. In particular, a heavy barbell was rested on his vertebral prominence at the level of C7/T1 during leg squatting. Repetitive trauma as a cause for extra-nuchal-type fibromas has been sparsely reported, but we suggest that sustained high pressure is an additional required feature. Although this lesion was in the posterior neck, it was contained entirely within the subcutaneous tissues without involvement of the nuchal ligament. Hence, it was considered an extra-nuchal fibroma. A description of key ultrasound and MRI imaging characteristics are provided to assist in making the diagnosis, along with a review of the current literature and a discussion of differential diagnoses.
\end{abstract}

Keywords Nuchal fibroma $\cdot$ Gardner $\cdot$ Repetitive trauma

\section{Introduction}

A nuchal-type fibroma is a rare, non-aggressive tumour, originally described as arising from the dorsocervical neck region. It was introduced as an entity by Enzinger and Weiss in 1988 [1] and was described as a rare benign fibrous proliferation characterised by hypocellular bundles of collagen interspersed with fatty tissue [2,3]. Since then, associations with diabetes mellitus, scleredema and Gardner's syndrome have emerged [2-6]. Trauma has been suggested as another cause [7]. While the majority of the tumours are found in the posterior neck, extra-nuchal sites have been less commonly reported $[2,3,8-10]$.

The authors identified only one other article on this condition within the radiologic literature [9]. This suggests limited knowledge of this condition amongst radiologists, who may be the first to reach the diagnosis based on the characteristic imaging findings.

Prem Ruben Jayaram

premruben@yahoo.com

1 Department of Radiology, Vancouver General Hospital, 899 W 12th Ave, Vancouver, BC V5Z 1M9, Canada

2 Department of Pathology, Vancouver General Hospital, 899 W 12th Ave, Vancouver, BC V5Z 1M9, Canada
This presentation is of the imaging features of an extranuchal-type fibroma. This lesion has been histologically confirmed. It was deemed extra-nuchal as it was entirely located in the subcutaneous fatty tissues without involvement of the nuchal ligament, and is the first reported case of an acquired fibroma in the gymnasium setting, secondary to high pressure repetitive trauma. The increasing popularity of this activity may lead to a rise in prevalence of this condition and the need for greater clinician and radiologist awareness.

\section{Case report}

A 34-year-old male presented to the orthopedics department having noticed an increasingly painful mass over his upper back and lower neck region along the midline. He stated that it had been growing slowly over a period of a few years.

Clinical examination revealed an approximately $5 \times 3 \mathrm{~cm}$ superficial solid immobile mass over the cervicothoracic junctional region along the midline. It was oriented elliptically along the craniocaudal axis but not tender to palpation and had no evidence of erythema. There was no restriction of movement or associated neurological symptoms. At this stage, a wide differential was considered, including nonaggressive entities such as a lipoma, desmoid fibromatosis, 
or myositis ossificans. Aggressive lesions were also considered but thought less likely.

An MRI scan was performed to characterise the mass. This demonstrated a poorly defined mass within the subcutaneous fatty tissues superficial to the $\mathrm{C} 7$ and $\mathrm{T} 1$ spinous processes measuring $4.7 \times 3.0 \times 1.8 \mathrm{~cm}$. It was low in $\mathrm{T} 1$ and T2 signal without any significant enhancement. High T1 and $\mathrm{T} 2$ signal fatty tissue was interspersed within this lesion in a limited streaky pattern (Figs. 1 and 2). The mass extended to the overlying dermis, which was mildly thickened. The reporting radiologist described this mass as an area of fibrosis on this MRI scan, without a demonstrable cause, and recommended a biopsy for further evaluation.

Given the signal characteristics, the differential diagnosis was rapidly narrowed to fibrous type lesions, as there was no osseous or cartilaginous area seen. This was not thought to represent a fibroma, which is typically well-circumscribed without interposition of fatty tissue. It is not in the typical location for an elastofibroma, which is known to occur deep to the serratus anterior and lattisimus dorsi muscles in the infrascapular region. It did not have the mass-like appearance, signal characteristics or enhancement pattern of desmoid fibromatosis. Consequently, the working diagnosis was that of bland non-specific fibrous proliferation, rather than a specific entity; a diagnosis of nuchal-type fibroma was not yet established.

An ultrasound-guided core biopsy was requested, at which time the procedural radiologist noticed that the patient had the physical appearance of a bodybuilder. The patient stated that he has been a regular gym attendee for at least a decade and his routine included heavy barbell squats with the bar across his upper shoulders at the site of the lesion. He stated that this had become increasingly difficult due to local tenderness, which would rapidly and spontaneously resolve within a couple of days after squats, suggestive of repetitive trauma to this site. Despite the increased discomfort, he had not changed his technique since he started doing these exercises, which he had been doing for many years prior to noticing this lesion.

Ultrasound demonstrated a $5.2 \times 3.2 \times 1.9 \mathrm{~cm}$ homogeneous hyperechoic ill-defined mass overlying the T1 spinous process and the skin without internal vascularity (Fig. 3). It is noteworthy that the biopsy was technically difficult to perform due to the very dense nature of the lesion, and a very large amount of force was required to penetrate the lesion with the biopsy needle. It also demonstrated a fibrous texture during needle penetration. It should be noted that the biopsy performed on this patient was technically challenging, as the dense nature of the mass made it particularly difficult to penetrate with a core biopsy needle. At this stage, given the new history, position, density and texture of the lesion, a diagnosis of nuchal-type fibroma was suggested.

Histopathological analysis from the biopsy sample showed features typical of a nuchal-type fibroma. These include thick bundles of haphazardly arranged collagen fibres with entrapment of adipose and nerve tissues (Fig. 4). Verhoeff-Van Gieson elastin stain was performed and showed a delicate network of normal elastic fibres (Fig. 5). A CT scan was not performed at any stage. The patient is currently awaiting resection of the nuchal-type fibroma, which has been delayed due to the COVID-19 pandemic.
Fig. 1 A T1 image of the cervical spine in the sagittal plane. B $\mathrm{T} 1$ with contrast enhancement. The nuchal-type fibroma overlies the $\mathrm{C} 7$ and $\mathrm{T} 1$ spinous processes and is marked with white arrows. The ligamentum nuchae is indicated with a white asterisk, and ends at the spinous process of C7. Note the lack of capsule and high $\mathrm{T} 1$ and $\mathrm{T} 2$ streaks, consistent with adipose tissue, interspersed within low signal regions, representing collagenous fibrous tissue

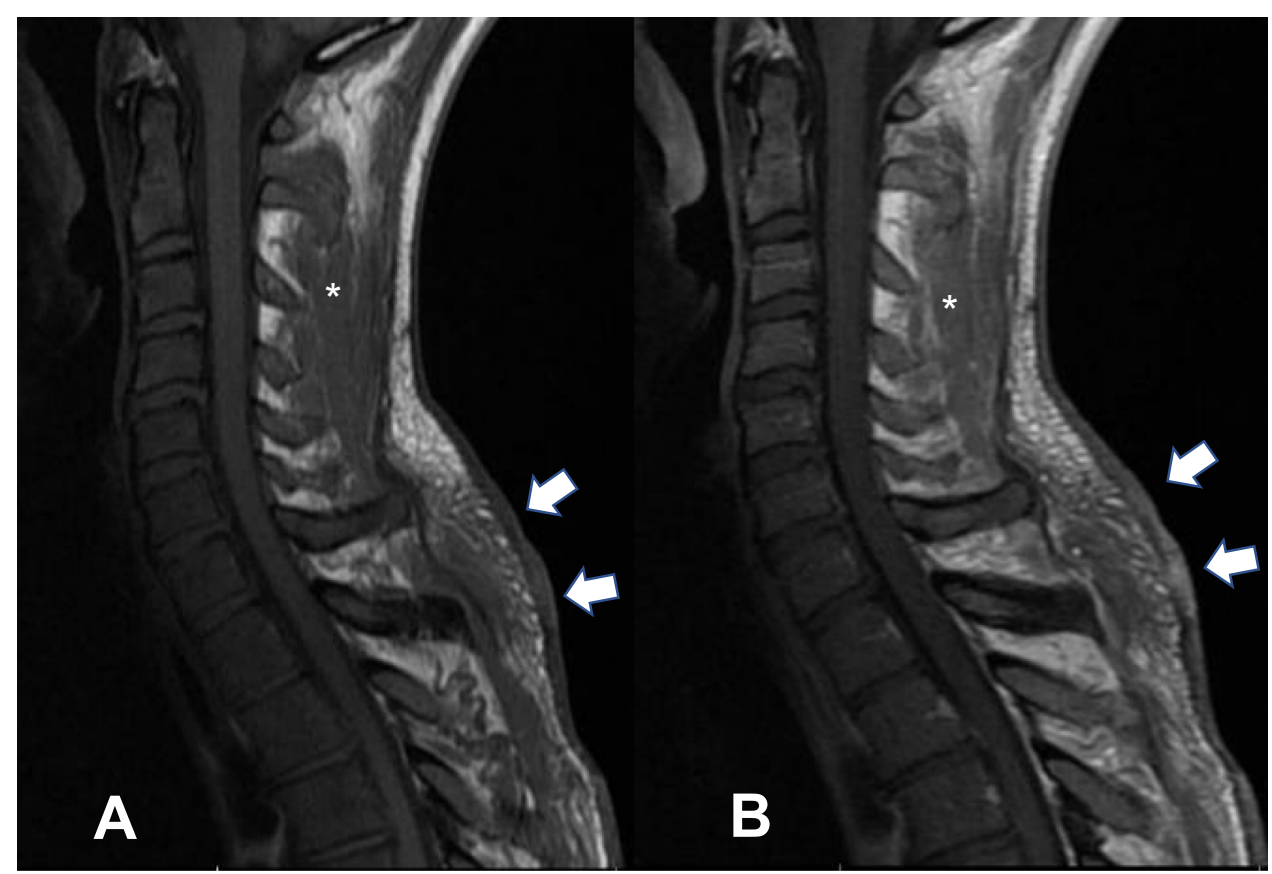




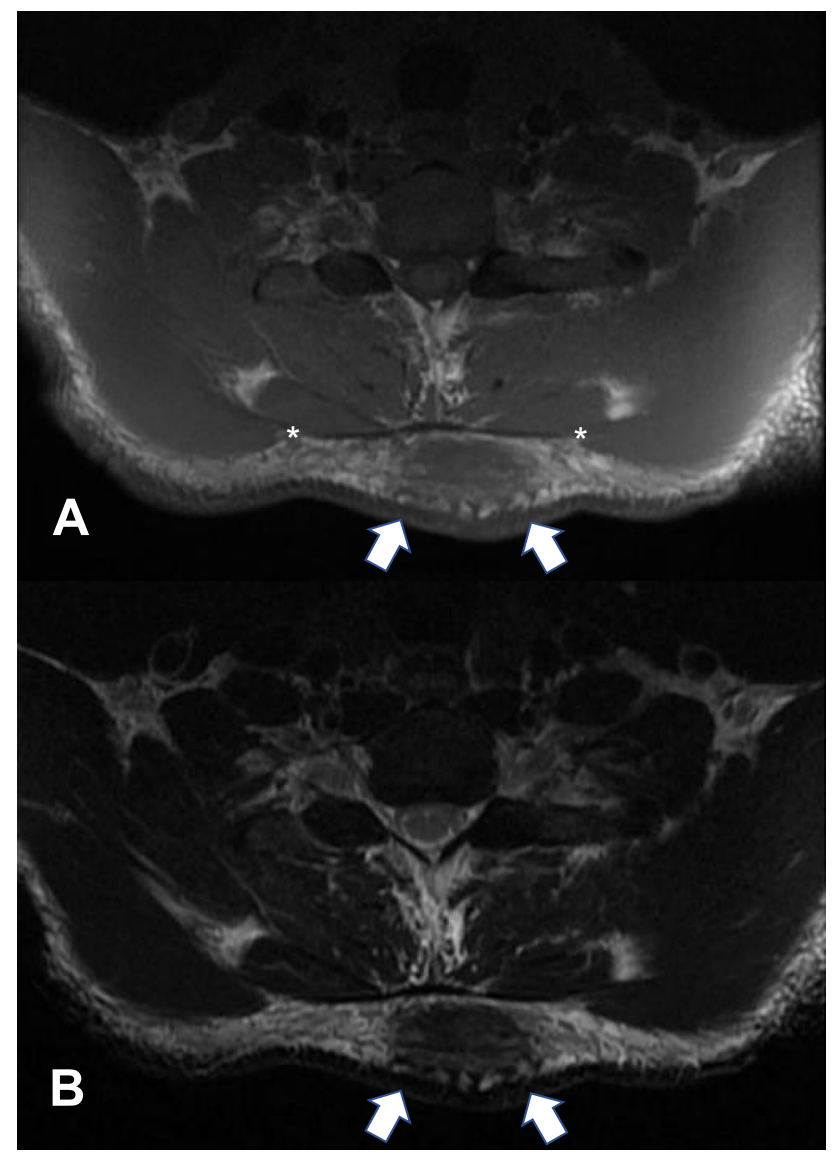

Fig. 2 A T2 axial image and B T1 axial image between the C7 and $\mathrm{T} 1$ spinous processes demonstrating the low signal nuchal-type fibroma in the subcutaneous tissues marked by white arrows extending up to the dermis. There is no evidence of invasion into the deeper tissues beneath the deep fascial layer, as indicated by the dark T1 line between the white asterisks

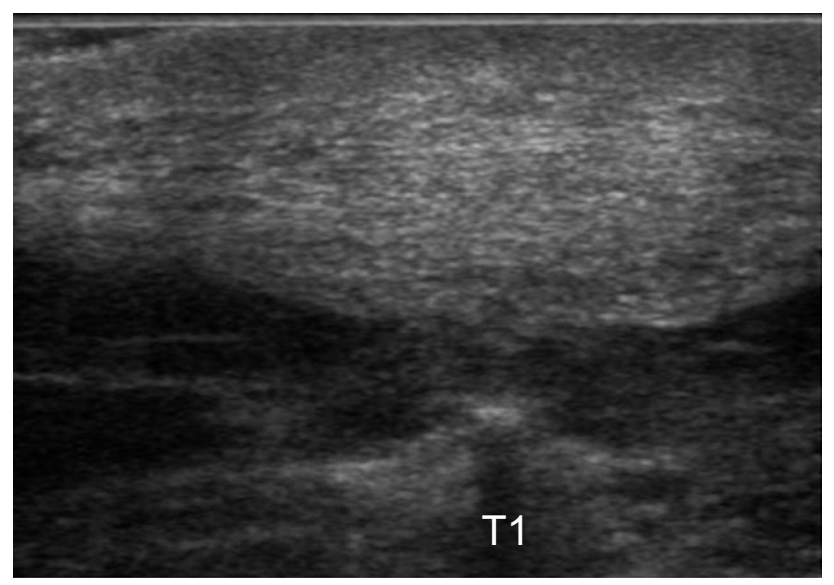

Fig. 3 Ultrasound image taken transverse across the level of the T1 spinous process. The mass is seen within the subcutaneous tissues but is poorly encapsulated. It demonstrates diffusely high echogenicity with slight heterogeneity. On Doppler imaging, no colour flow was seen

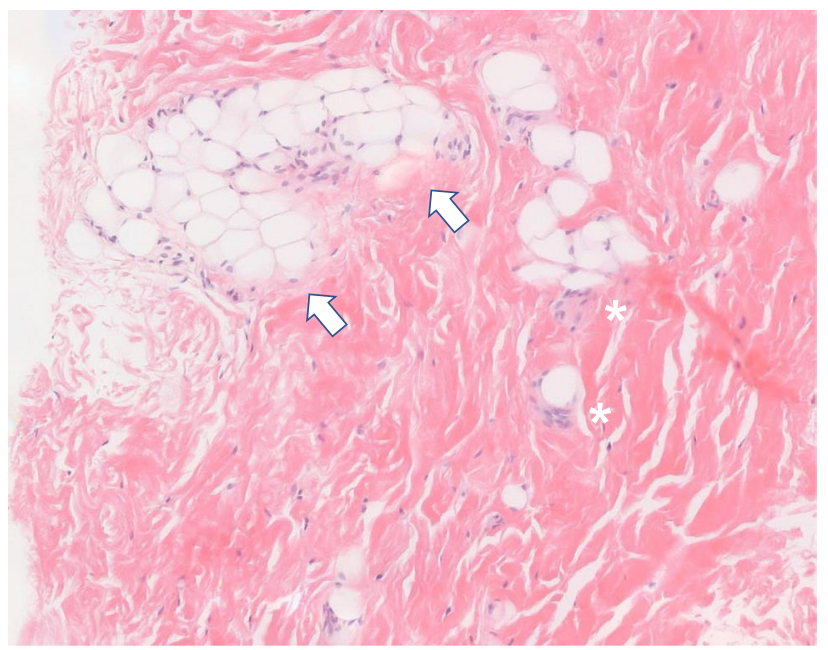

Fig. 4 Core biopsy sample, H\&E stain. Note the thick bundles of haphazardly organised collagen fibres, encasing the lobulated adipose tissue (arrows), and relative scarcity of nuclei (asterisks). Nerve and skeletal muscle involvement is not seen in this specimen

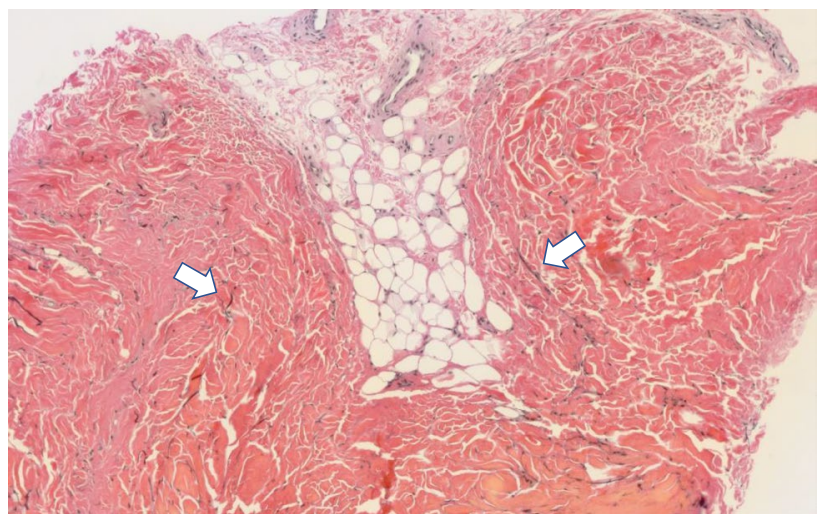

Fig. 5 Core biopsy sample, Verhoeff-Van Gieson elastin stain. A delicate network of black-stained elastic fibres was seen between the collagen fibres (arrows), which comprise the majority of the sampled tissue

\section{Discussion}

Nuchal-type fibromas typically present as an asymptomatic, superficial slowly growing mass. Clinically, these are typically palpable, non-tender, non-mobile and firm. There is a wide age range at presentation between 2 and 74 years, with a 4 to 1 male to female predilection [2]. There may or may not be erythema over the mass, especially in cases of recent low-grade trauma. Common locations for extra-nuchal involvement include the face, back and shoulders $[2,10]$. The largest mass was demonstrated to be up to $20 \mathrm{~cm}$ in the shoulder region [11]. The treatment for these benign masses is resection, although they 
have a tendency to recur, possibly due to the continued presence of a stressor, such as repetitive trauma $[2,10]$.

Microscopically, nuchal-type fibromas have been described as hypocellular, haphazardly arranged dense collagenous matrix with entrapped adipose tissues and nerves, and scattered fibroblasts [2, 3, 5-8]. In certain cases, mucoid degeneration may be seen [12]. In the largest reported series by Michel et al., $44 \%$ of patients had diabetes [2] and several reports have emerged of nuchal-type fibromas occurring in patients with Gardner's syndrome [2-6, 12]. These can be identical to Gardner-type fibromas, and if detected in the pediatric population, in multiple or unusual locations, investigating for Gardner's syndrome may be prudent [12].

This case represents a nuchal-type fibroma occurring ectopic to the ligamentum nuchae, thought to be as a consequence of repetitive trauma. Only one case series has previously reported this phenomenon, involving palanquin bearers in Korea [7]. In depth review of the literature reveals fewer than 100 cases, which is interesting as one would expect the prevalence of repetitive trauma-induced nuchaltype fibroma to be much greater.

Both repetitive trauma and lifting heavy burdens are common occurrences; certainly, this is so in the gym. The authors postulate that sustained high pressure on an atypical body area may be also required for this lesion to develop. For example, despite sitting or walking for long hours, or performing heavy lifting activities with a person's hands, these lesions are extremely uncommon at these sites. During the history, this patient admitted resting the barbell along his vertebral prominence, as opposed to over his trapezius muscles, which focuses pressure on normally non-weight bearing skin. The latter is also considered proper technique and would be naturally adopted by most weightlifters to reduce pressure-induced discomfort. The patients in the Korean study were unable to adopt pressure-relieving positions or techniques due to the nature of their work, consistent with this hypothesis.

An ultrasound image is provided from our case, which has not been previously published to the best of our knowledge. Oftentimes the lesion may be too large to view in a single ultrasound image which typically shows a poorly encapsulated hyperechogenic area. The high echogenicity is most likely due to the interspersed fat. Minimal to no Doppler signal is seen within the lesion. These twin features of no internal vascularity and intralesional fat are strongly suggestive of a non-aggressive process

At this juncture, if other imaging is unavailable, it may be reasonable to conclude that this is a non-aggressive lesion which can be monitored clinically, even if the specific diagnosis is not reached. A follow-up ultrasound scan could also be considered to confirm stability. On occasion though, it can be inseparable from the dermis or infiltration of skeletal muscle can occur $[2,10]$. These features were not demonstrated in this case, but would warrant further investigation.

If only a single diagnostic test were to be ordered, an MRI scan is the investigation of choice, with or without contrast. The mass is poorly defined and demonstrates predominantly low T1 and T2 signal as a consequence of low water content and high density of fibrous tissue. In larger lesions, high T1 and $\mathrm{T} 2$ signal regions can be seen within interspersed within low signal regions in a streak-like fashion due to the presence of entrapped adipose tissue. This intralesional fat again indicates a non-aggressive process. Minimal enhancement is seen on contrast administration due to the relative hypocellular nature of nuchal-type fibromas [8, 10, 12].

Of the relatively few cases reported in the literature, we are only aware of one case report in a radiology journal. This paper demonstrates mild enhancement of the lesion [9]. We differ from this report in that no enhancement was demonstrated when gadolinium was given for our case, in line with most other reports, likely due to the hypocellular nature of the mass.

CT scans are not considered to be useful in the evaluation of these lesions due to the relative lack of enhancement and contrast with surrounding tissues, and lack of specificity in diagnosis [5].

Given the difficulty in penetrating the lesion during the ultrasound-guided biopsy, caution is advised for operators, especially if there are critical structures beyond the mass.

It is relatively straightforward to differentiate nuchal-type fibromas from malignant entities such as malignant fibrous histiocytomas, fibrosarcomas and liposarcomas due to their relatively low vascularity, slow-growing natural history, or lack of enhancement and invasive features [2, 7, 8]. Larger nuchal-type fibromas can be easily distinguished from other fibromas due to their high echogenicity and intralesional fat, which tend not to have these characteristics. If there is ambiguity, especially for smaller lesions without characteristic imaging findings, microscopic and histological analyses from biopsy samples are usually conclusive.

Desmoid-type fibromas typically involve deep soft tissues, have more prominent vascularity, and stain positive for beta-catenin histologically. Elastofibromas and fibromas of the tendon sheath can usually be excluded based on location, occurring in the infrascapular chest wall and distal extremities respectively [2, 3, 7-9, 13, 14]. Desmoplastic fibroblastomas (collagenous fibromas) sometimes demonstrate heterogeneous enhancement but can otherwise be indistinguishable from nuchal-type fibromas on imaging [13]. Histologically they are distinct, with elastofibromas consisting of fibrocollagenous proliferation with abnormal elastic fibres present between collagen bundles. The elastic fibres are eosinophilic, thick, fragmented, and globular resembling "beads on a string". In comparison, nuchal-type fibroma shows thick collagen bundles with delicate elastic 
fibres. Desmoplastic fibroblastomas do not stain for CD34, whereas nuchal-type fibromas are diffusely positive [6, 7, $9,15,16]$.

En bloc resection of the tumour is the treatment of choice, although recurrence is a recognised complication [2]. This is possibly due to incomplete margins, with surgery made more difficult due to the unencapsulated and poorly defined nature of the lesion, especially if it involves skeletal muscles or other deep tissue structures. Re-excision remains the best option if clinically warranted.

In conclusion, this case illustrates that extra-nuchal fibromas may be induced by repetitive trauma and that high pressure in a body part not typically used for such load bearing may be a contributing factor. This is a relatively rare entity and the first case described as a result of weightlifting exercises in the gym, which is a growing activity worldwide.

\section{Declarations}

Conflict of interest The authors declare no competing interests.

\section{References}

1. Enzinger FM, Weiss SW. Benign tumors and tumorlike lesions of the fibrous tissue. $2^{\text {nd }}$ C.V Mosby, St. Louis, MO, 1988 102-135

2. Michal M, Fetsch JF, Hes O, Miettinen M. Nuchal-type fibroma. Cancer. 1999;85(1):156-63.

3. Lee SE, Kim YC, Kim S-C. Nuchal fibroma presenting as two posterior neck masses. J Dermatol. 2007;34(4):262-3.

4. Banney LA, Weedon D, Muir JB. Nuchal fibroma associated with scleredema, diabetes mellitus and organic solvent exposure. Aust J Dermatol. 2000;41(1):39-41.

5. Dawes LC, La Hei ER, Tobias V, Kern I, Stening W. Nuchal fibroma should be recognized as a new extracolonic manifestation of Gardner variant familial adenomatous polyposis. Australian and New Zealand J Surg. 2000;70:824-6.

6. Diwan AH, Graves ED, King JA, Horenstein MG. Nuchal-type fibroma in two related patients with Gardner's syndrome. Am J Surg Pathol. 2000;24:1563-7.

7. Lee CC, Lai CS, Lin CH, et al. Extra nuchal-type fibroma associated with repetitive blunt trauma during religious activities. Trauma Case Rep. 2016;4:16-20.

8. Sraj SA, Lahoud LE, Musharafieh R, Taha A. Nuchal-type fibroma of the ankle: a case report. J Foot Ankle Surg. 2008;47(4):332-6.

9. Lee GK, Suh KJ, Lee SM, Lee SJ. Nuchal-type fibroma of the buttock: magnetic resonance imaging findings. Jpn J Radiol. 2010;28:538-41.

10 Hameed M, Benevenia J, Blacksin M, Aisner SC. Nuchal fibroma of the shoulder involving skeletal muscle: a radiographic and clinicopathological study. A case report. J Bone Jt Surg. 1998;80:1684-6.

11. Gong Y, Zhao X, Wu D, Liu J. Nuchal-type fibroma of the shoulder: a case report and review of the literature. Oncol Lett. 2016;11(6):4152-4.

12. Karonidis A, Rigby HS, Orlando A. Collagenosis nuchae: a case report of a rare and often misdiagnosed condition. J Plast Reconstr Aesthet Surg. 2007;60(3):320-3.

13. Kamata Y, Anazawa U, Morioka H, et al. Natural evolution of desmoplastic fibroblastoma on magnetic resonance imaging: a case report. J Med Case Rep. 2011;5:139.

14. Dinauer PA, Brixey CJ, Moncur JT, Fanburg-Smith JC, Murphey MD. Pathologic and MR imaging features of benign fibrous softtissue tumors in adults. Radiographics. 2007;27(1):173-87.

15. Singh NG, Mannan AASR, Kahvic M. Desmoplastic fibroblastoma (collagenous fibroma): report of a case. Indian J Pathol Microbiol. 2011;54(1):206-7.

16. Michal Z, Michal M. Nuchal-type fibroma is positive for CD34 and CD99. American J Surg Pathol. 2001;25(7):970.

Publisher's note Springer Nature remains neutral with regard to jurisdictional claims in published maps and institutional affiliations. 